\title{
Screening Nuclear Field Fluctuations in Quantum Dots for Indistinguishable Photon Generation
}

\author{
R. N. E. Malein, ${ }^{1}$ T. S. Santana, ${ }^{1}$ J. M. Zajac, ${ }^{1}$ A. C. Dada, ${ }^{1}$ E. M. Gauger, ${ }^{1}$ P. M. Petroff, ${ }^{2}$ \\ J. Y. Lim, ${ }^{3}$ J. D. Song, ${ }^{3}$ and B. D. Gerardot ${ }^{1, *}$ \\ ${ }^{1}$ SUPA, Institute of Photonics and Quantum Sciences, Heriot-Watt University, Edinburgh EH14 4AS, United Kingdom \\ ${ }^{2}$ Materials Department, University of California, Santa Barbara, California 93106, USA \\ ${ }^{3}$ Center for Opto-Electronic Convergence Systems, KIST, Seoul 136-791, Republic of Korea
}

(Received 31 December 2015; published 24 June 2016)

\begin{abstract}
A semiconductor quantum dot can generate highly coherent and indistinguishable single photons. However, intrinsic semiconductor dephasing mechanisms can reduce the visibility of two-photon interference. For an electron in a quantum dot, a fundamental dephasing process is the hyperfine interaction with the nuclear spin bath. Here, we directly probe the consequence of the fluctuating nuclear spins on the elastic and inelastic scattered photon spectra from a resident electron in a single dot. We find the in-plane component of the nuclear Overhauser field leads to detuned Raman scattered photons, broadened over experimental time scales by field fluctuations, which are distinguishable from both the elastic and incoherent components of the resonance fluorescence. This significantly reduces two-photon interference visibility. However, we demonstrate successful screening of the nuclear spin noise, which enables the generation of coherent single photons that exhibit high visibility two-photon interference.
\end{abstract}

DOI: 10.1103/PhysRevLett.116.257401

Indistinguishable single photons are an essential resource for quantum photonic logic gates and networking [1]. Among the various approaches for generating identical light quanta, resonance fluorescence (RF) from a semiconductor quantum dot (QD) [2-10] is one of the most promising for practical technological implementation. The RF spectrum is composed of elastic and inelastic scattered light [11-14]. The elastic or Rayleigh scattered light, first measured in homodyne absorption experiments on a QD [15], has the first-order coherence properties of the laser but the second-order coherence properties of the emitter [16]. This light, which can be imprinted with an arbitrary phase or temporal profile [3], is fundamentally indistinguishable. $\mathrm{RF}$ can therefore relax the requirement for a perfectly stable, transform-limited optical transition that is difficult to realize in the solid state. Specifically, compared to nonresonant excitation followed by spontaneous emission, RF helps overcome the relatively slow spectral fluctuations caused by charge noise in the QD environment [14,17].

In addition to the elastic and incoherent components of RF, near resonant excitation can lead to Raman scattering into another ground state. Taken in isolation, Raman scattering from a QD with a resident electron or hole can have several attractive features. First, below saturation, the coherence of the Raman photons is determined by the ground-state dephasing rather than that of the excited state [18-20].

Published by the American Physical Society under the terms of the Creative Commons Attribution 3.0 License. Further distribution of this work must maintain attribution to the author(s) and the published article's title, journal citation, and DOI.
Second, Raman photons are highly tunable: their energy is determined by the detuning of the driving field rather than the fundamental optical transition energy [18,20,21]. Finally, because of clean selection rules in QDs, the polarization of the Raman scattered photons can be linked to the spin of the final state [20-22], enabling spin-photon entanglement [23-25] and raising the prospect for quantum networks [26]. Typically, spin-flip Raman photons are generated using an in-plane external magnetic field $\left(B_{\text {ext }}^{\|}\right)$to mix the spin states [27]. To take advantage of these features, $B_{\text {ext }}^{\|}$must be large enough that the spin-flip Raman scattering can easily be distinguished and filtered from the elastic and incoherent components [18-21]. By contrast, our goal in this Letter is to maximize Rayleigh (as opposed to Raman) scattering, as will be explained in the following.

A III-V QD also contains a modest intrinsic effective magnetic field, the Overhauser field $\left(B_{N}\right)$, due to the coupling of the QD's constituent atoms' nuclear spins with the electron spin via the contact part of the hyperfine interaction. Incomplete cancellation of the finite random nuclear spin orientations leads to an effective field $B_{N} \approx 10-30 \mathrm{mT}$ that fluctuates on a $10^{-4} \mathrm{~s}$ time scale $[17,28,29]$. The electron spin precession around $B_{N}$ leads to ensemble dephasing on nanosecond time scales [28-30]. The fluctuating arbitrary orientation of $B_{N}$ also leads to an almost always present inplane component $B_{N}^{\|}$which affects the optical properties of a quantum dot. Kuhlmann et al. have investigated the role of nuclear spin noise on the neutral $\left(X^{0}\right)$ and negatively charged $\left(X^{1-}\right)$ states of a QD [31], while Hansom et al. recently exploited $B_{N}^{\|}$to achieve coherent control and two-color coherent population trapping with $X^{1-}$ [32]. Here, we report 
new insights on the effect of $B_{N}^{\|}$on the RF spectrum of a single QD and its consequences on indistinguishable photon generation. We find that spin mixing causes Raman scattered photons clearly distinguishable from the elastic spectrum that reduce the visibility $(\nu)$ of two-photon interference (TPI) for $X^{1-}$. We demonstrate the screening of $B_{N}^{\|}$for both $X^{0}$ and $X^{1-}$ via either an effective magnetic field due to an exchange interaction [33] or a perpendicular external magnetic field $\left(B_{\text {ext }}^{\perp}\right)$. In each case, the spectra exhibit near-ideal two-level behavior and ultrahigh visibility TPI is achieved.

The self-assembled InGaAs QDs studied in this work were embedded in a GaAs Schottky diode for deterministic charge state control. Numerous QDs from two samples at $T=4 \mathrm{~K}$ were investigated and provided consistent results. Sample 1, previously used in Ref. [34], yields $\approx 350 \mathrm{kHz}$ from a single QD on a single photon detector at saturation in cw RF. Sample 2 has the identical Schottky diode design, but the QDs are positioned at an antinode of a fifth-order planar cavity on top of a Au layer which functions as a mirror and Schottky gate. Sample 2 yields $\approx 10$ times higher count rates due to the planar cavity design [35]. The RF, obtained using orthogonal linear polarizers in the excitation and collection arms of a confocal microscope $[3,4,13,17]$, is characterized by three techniques: high-resolution (27 MHz) spectroscopy using a Fabry-Perot interferometer (5.5 GHz free spectral range), a Hanbury Brown-Twiss interferometer to measure $g^{2}(\tau)$, and an unbalanced Mach-Zender (MZ) interferometer $\left(\Delta t_{\text {delay }}=49.7 \mathrm{~ns}\right)$ with polarization control in each arm to measure postselected, two-photon interference [2,6]. In the MZ setup, the beam splitters have nearly perfect 50:50 reflection-totransmission ratio. For TPI experiments, a signal from a single QD excited with a cw laser is used, with the delay line in the MZ incorporated to overcome the QD emission's antibunched character and allow two photons to be incident on the second beam splitter simultaneously. A grating (bandwidth $f / 2 \pi \sim 1.9 \mathrm{GHz}$ ) is used to spectrally filter the zero-phonon line from the majority of the phonon sideband.

Figure 1 reveals our main spectroscopic result. Here, we compare the resonantly scattered zero-phonon photon spectra from three scenarios: $X^{0}$ and $X^{1-}$ with $B_{\text {ext }}=0 \mathrm{~T}$, and $X^{1-}$ with $B_{\text {ext }}^{\perp}=0.6 \mathrm{~T}$. The spectra are recorded over a range of Rabi frequencies $(\Omega)$, from $\Omega \ll \Omega_{S}$, where coherent scattering dominates, to $\Omega \gg \Omega_{S}$, where incoherent emission dominates. Here, the saturation parameter $\Omega_{S}$ is given by $\sqrt{1 /\left(T_{1} T_{2}\right)}$, where $T_{1}$ is the lifetime and $T_{2}$ the dephasing time. Figure 1(c) shows that near-ideal elastic and inelastic spectra are observed for $X^{0}$, although for $\Omega \gg \Omega_{S}$ the sidebands of the Mollow triplet are slightly broadened from Mollow's theory due to a modest amount of slow spectral fluctuations (which do not lead to pure dephasing) [14]. The central incoherent Lorentzian linewidth is measured as $318 \pm$ 9.3 $\mathrm{MHz}$ (FWHM), closely matching the Fourier transform limit of the measured $T_{1}$ value of $550 \pm 40$ ps. Crucially, when the population of the excited state is minimal $\left(\Omega \ll \Omega_{S}\right)$, the elastic component dominates the $X^{0}$ spectrum, confirming minimal pure dephasing. In Fig. 1(b) we plot the ratio of the elastic component to the total spectrum (ER). The solid line is a fit given by $[11,36]$
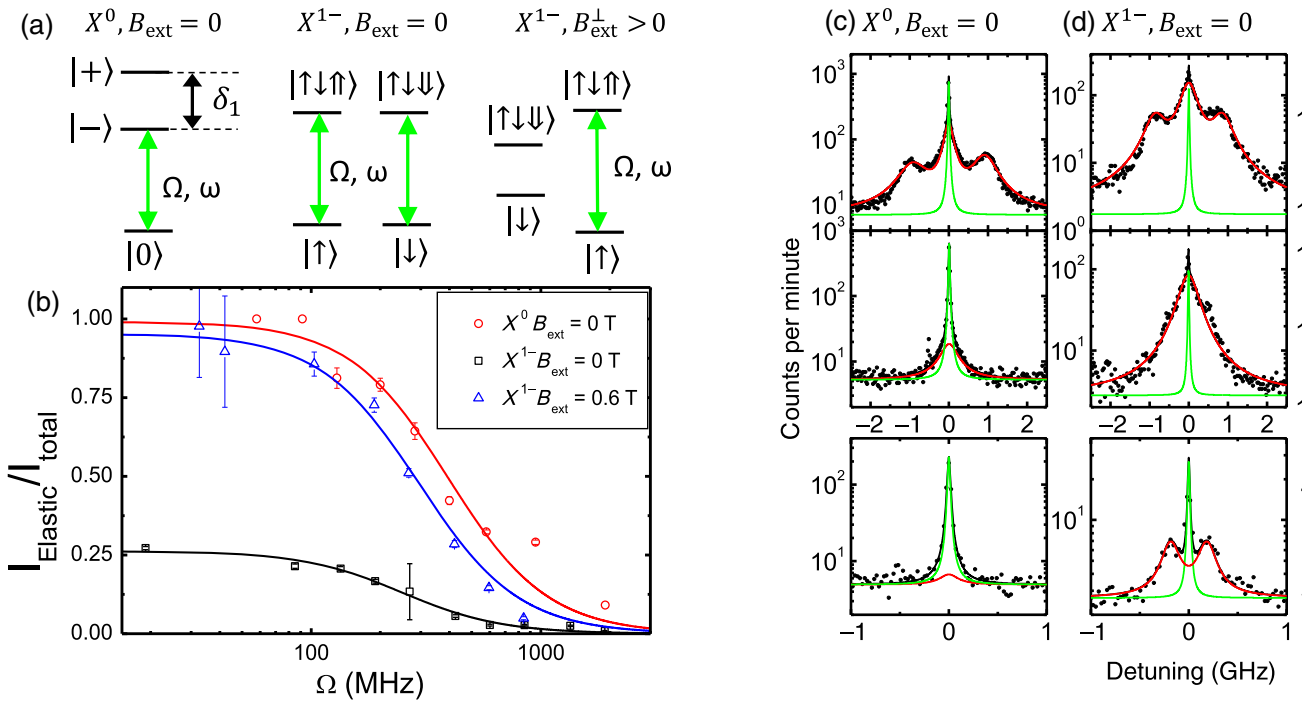

(e) $X^{1-}, B_{\text {ext }}^{\perp}=0.6 \mathrm{~T}$

FIG. 1. (Left panels) (a) Level diagrams for $X^{0} ; X^{1-}$ at $B_{\text {ext }}=0 \mathrm{~T}$; and $X^{1-}$ at $B_{\text {ext }}^{\perp}>0 \mathrm{~T}$, where $|+\rangle$ and $|-\rangle$ are the symmetric and antisymmetric neutral exciton eigenstates, respectively, $\delta_{1}$ is the splitting due to the anisotropic part of the electron-hole exchange interaction, and the green arrows represent the driving fields. (b) Plot of the ratio of elastic scattered photons to the total intensity. The points are based on the fits to the RF spectra and the curve is a fit using Eq. (1). (Right panels) High-resolution spectra of (c) $X^{0}$, (d) $X^{1-}$ at $B_{\mathrm{ext}}^{\perp}=0 \mathrm{~T}$, and (e) $X^{1-}$ at $B_{\text {ext }}^{\perp}=0.6 \mathrm{~T}$, above $\left(\Omega>\Omega_{S}\right)$, near $\left(\Omega \approx \Omega_{S}\right)$, and below $\left(\Omega<\Omega_{S}\right)$ the saturation Rabi frequency. The green (red) line is a single (multiple) Lorentzian fit to the elastic (inelastic) component of the RF. The black curve is the total Lorentzian fit. Data are taken from sample 1. 


$$
E R=\frac{T_{2}^{*}}{2 T_{1}} \frac{1}{1+\Omega^{2} / \Omega_{S}^{2}},
$$

where $T_{2}^{*}$ is the ensemble dephasing time obtained in timeaveraged spectra. By fitting the complete data set, we determine the only unknown parameter, $T_{2}^{*}$, accurately and we find $T_{2}^{*}=(1.96 \pm 0.08) T_{1}=1.08 \pm 0.09 \mathrm{~ns}$ for $X^{0}$.

The spectra for $X^{1-}$ with $B_{\text {ext }}=0 \mathrm{~T}$ in Fig. 1(d) deviate significantly from the near-ideal two-level behavior exhibited by $X^{0}$. All $X^{1-}$ spectra show a reduced elastically scattered component compared to $X^{0}$. Additionally, a doublet surrounding the elastic peak is clearly evident. For $\Omega \ll \Omega_{S}$, the doublet is separated by $\approx 190 \mathrm{MHz}$ and each peak in the doublet has a linewidth of $\approx 210 \mathrm{MHz}$. Figure $1(b)$ shows that the ER saturates at only $\approx 26 \%$ and the fit yields $T_{2}^{*}=(0.52 \pm 0.02) T_{1}=0.40 \pm 0.07 \mathrm{~ns}$. However, the near-ideal two-level behavior can be recovered with a modest magnetic field in the growth direction, as shown in Fig. 1(e) for $B_{\mathrm{ext}}^{\|}=0.6 \mathrm{~T}$. Based on the fit to the ER, $T_{2}^{*}=(1.94 \pm 0.01) T_{1}=1.51 \pm 0.07 \mathrm{~ns}$.

To investigate the origin of the sidebands for $X^{1-}$, we characterize the RF spectrum as a function of laser detuning $\Delta_{L}$ for $\Omega \ll \Omega_{S}$. Here, we observe that the entire spectrum follows $\Delta_{L}$ [Fig. 2(a)], and the integrated intensity of the spectrum exhibits a Lorentzian line shape with a linewidth of $\approx 850 \mathrm{MHz}$ [Fig. 2(b)]. This observation eliminates the possible presence of incoherent emission in the spectrum, which is independent of laser detuning. We therefore identify the central peak as the elastic component and the doublet as spin-flip Raman scattering. A schematic of the effect is shown in Fig. 2(d), in which we depict the fourlevel system for two values of the $B_{N}^{\|}$component which split the ground-state electron spin states. We assume degenerate excited states, as the hyperfine interaction for hole spins is significantly reduced compared to the electron for $B_{N}^{\|}[37,38]$. The solid green arrows represent the driving field on resonance with the unperturbed (e.g., $B_{N}^{\|}=0 \mathrm{~T}$ ) transitions. This driving field determines the elastic scattering spectrum. The red and blue dashed lines represent redand blue-Raman scattering detuned by $2 \mu_{B} g_{e} B_{N}^{\|}=\Delta E_{Z}$. The linewidth of these photons is inversely proportional to the incoherent spin-flip time. The experimental measurement time of a few minutes for each spectrum signifies a time-averaged measurement of $\approx 10^{5}$ orientations of $B_{N}$, effectively broadening the observed Raman sidebands according to the variance $\delta B_{N}^{\|}$. Notably, we also observe QDs which exhibit sidebands with a smaller detuning than in Fig. 2(a) that can be caused by either reduced nuclear spin fluctuations or $g_{e}^{\|}$. Further details on numerical simulations of the resonantly scattered power spectrum based on the optical Bloch equations of the four-level system with a linearly polarized driving field are provided in the Supplemental Material [39]. Numerical simulations to fit the experimental spectra yield a mean energy of $g \mu_{B} B_{N}=264 \pm 113 \mathrm{MHz}$, with a standard deviation
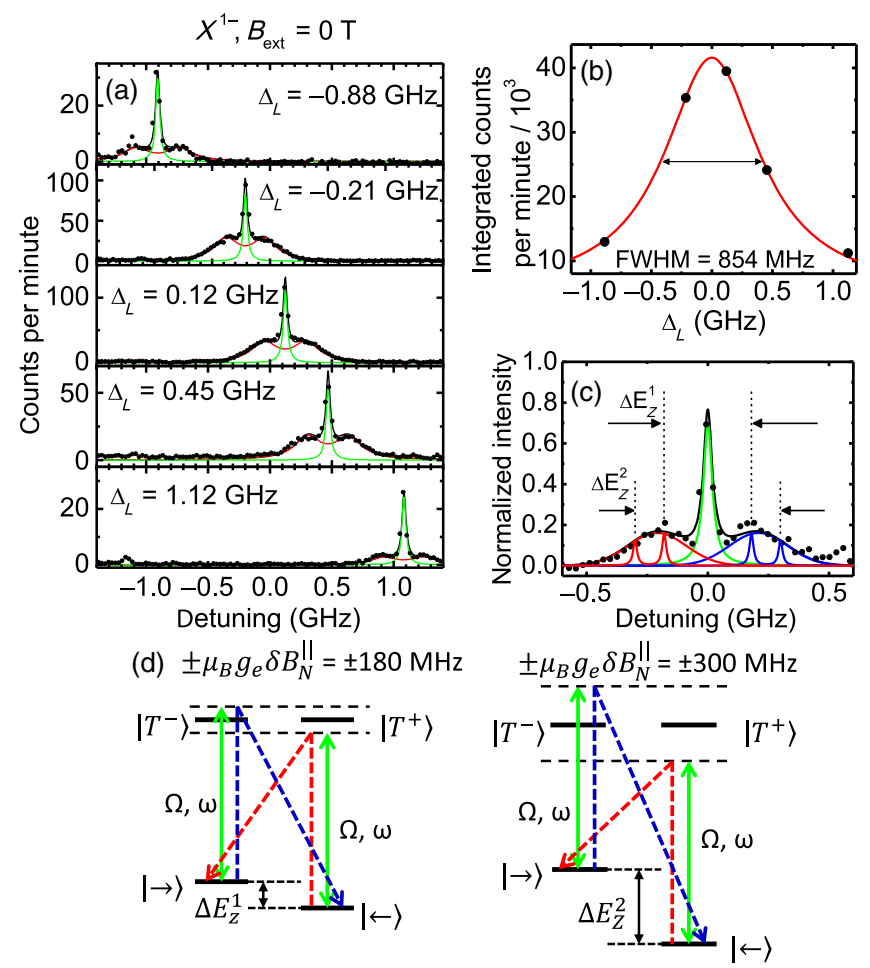

FIG. 2. (a) High-resolution spectra as a function of laser detuning $\left(\Delta_{L}\right)$ demonstrate that the central peak and sidebands are dependent on the laser energy. (b) The intensity of the detuned spectra reveal a Lorentzian line shape (the red curve). (c) A simulated spectrum based on the four-level model including $B_{N}$ (the black curve) fit to the experimental data (the black points) for $\Omega=0.25 \Omega_{S}$. The green line represents elastically scattered photons. The red and blue lines represent the red- and blue-detuned Raman photons as shown in the level diagrams for two different $B_{N}^{\|}$ values. Data are from sample 1. (d) Level diagrams for two different values of $B_{N}^{\|}$. For constant excitation energy, changes in $B_{N}^{\|}$change the splitting of the Raman transitions from the laser: fluctuations in the Overhauser field result in broadened Raman sidebands.

of $g \mu_{B} \delta B_{N}$ of $120 \pm 6 \mathrm{MHz}$. The black curve in Fig. 2(c) shows this simulation. Assuming a typical isotropic electron $g$ factor of -0.6 corresponds to a mean Overhauser field of $B_{N}=31 \pm 13 \mathrm{mT}$ and $\delta B_{N}=14 \pm 1 \mathrm{mT}$.

As the Overhauser field is ubiquitous in III-V QDs, the spectra from both $X^{1-}$ with a modest $B_{\mathrm{ext}}^{\perp}$ and $X^{0}$ demonstrate successful screening of the nuclear spin fluctuations. In the case of $X^{0}$, the anisotropic exchange interaction $\left(\delta_{1}\right)$ generates a fine-structure splitting of $\approx 2.4 \mathrm{GHz}$. In addition, the symmetric part of the exchange interaction energy is $\sim 70 \mathrm{GHz}$ [33]. Both are much larger than $\mu_{B} g_{e} B_{N}^{\|}=264 \mathrm{MHz}$. In the case of $B_{\mathrm{ext}}^{\perp} \gg B_{N}$ for $X^{1-}$, $B_{\text {ext }}^{\perp}$ stabilizes the electron spin which then precesses around $B_{\mathrm{tot}}=B_{\mathrm{ext}}^{\perp}+B_{N}$ [30]. This significantly reduces the branching ratio for spin-flip scattering, which can be estimated by $\left(B_{N}\right)^{2} /\left(2 B_{\mathrm{ext}}^{\perp}{ }^{2}\right)$ [45]. Hence, the nuclear spin fluctuations are effectively screened and Raman scattered photons are not visible in the spectra of Fig. 1(e). 

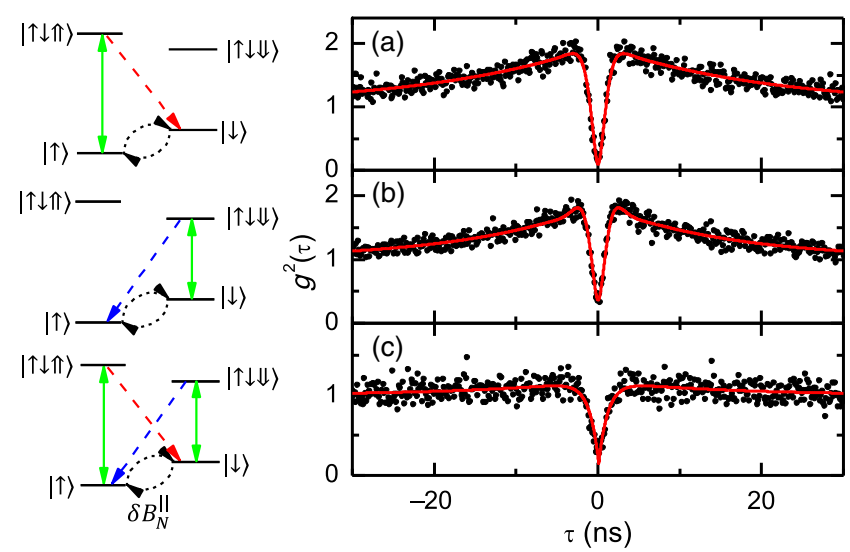

FIG. 3. Second-order correlation measurements on the Zeeman-split $X^{1-}$ transitions at $B_{\mathrm{ext}}^{\perp}=0.6 \mathrm{~T}$ and $\Omega=0.5 \Omega_{S}$. Single laser excitation of the (a) blue- and (b) redshifted lines show bunching around $\tau=0$, with decay time $\sim 20 \mathrm{~ns}$. Simultaneous two-laser excitation (c) shows suppressed bunching, demonstrating that its origin is spin pumping. Data are from sample 1.

As a precursor to TPI experiments, we first investigate the effect of $B_{N}$ on the second-order coherence $\left[g^{2}(\tau)\right]$. Figure 4(a) shows the autocorrelation from $X^{0}$ with a raw value of $g^{2}(0)=0.20 \pm 0.02$, limited by detector jitter. Upon deconvolution of the detector response, $g^{2}(0) \rightarrow 0$ (see Sec. II of the Supplemental Material [39] for details on the deconvolution). For $X^{1-}$, while near-ideal antibunching is again demonstrated, significant bunching around the $\tau=0$ dip is observed at $B_{\text {ext }}^{\perp}=0.6 \mathrm{~T}$ [see Figs. 3(a) and 3(b)] due to optical spin pumping caused by electron spin mixing $[27,32,45]$. The bunching amplitude is determined by the spin pumping fidelity, and the decay of the bunching is determined by the spin-relaxation dynamics. A two-color excitation scheme can be used to frustrate the spin pumping mechanism, which unambiguously demonstrates that the nature of the bunching is spin initialization
[Fig. 3(c)]. Although we have observed small bunching amplitudes even at $B_{\text {ext }}=0 \mathrm{~T}$ for a few QDs, it is typically negligible for on resonance driving, as in Fig. 4(b).

The canonical test for photon indistinguishability is twophoton interference, and the visibility is defined as $\nu=\left[g_{\perp}^{2}(0)-g_{\|}^{2}(0)\right] / g_{\perp}^{2}(0)$, where $g_{\perp}^{2}(0)$ and $g_{\|}^{2}(0)$ use orthogonal and parallel linear polarizations, respectively, in the two MZ arms. TPI measurements from $X^{0}$ at $\Omega=0.5 \Omega_{S}$ [Fig. 4(a)] reveal near perfect photon indistinguishability, with a deconvolved (raw) fit value of $\nu=0.99 \pm 0.02(\nu=0.58 \pm 0.04)$. This impressive result is expected, based on the near-ideal high-resolution spectrum, confirming that $\delta_{0}$ screens $B_{N}$ for $X^{0}$. In contrast, the same measurement on the $X^{1-}$ with $B_{\text {ext }}=0$ T [Fig. 4(b)] shows a reduced $\nu$ : a deconvolved (raw) fit value of $\nu=$ $0.67 \pm 0.04(\nu=0.50 \pm 0.05)$ is measured. Nevertheless, a high degree of indistinguishability is still observed, which is somewhat surprising considering the spectra in Fig. 1(d). However, in the "frozen-fluctuation" model, $B_{N}$ is static within the $\Delta t_{\text {delay }}=49.7 \mathrm{~ns}$, signifying that only one spinflip Raman transition energy is relevant for TPI. In other words, only narrow-band Raman photons will accompany the elastic and modest incoherent components of the RF spectrum over this time scale at $\Omega=0.5 \Omega_{S}$. Finally, applying $B_{\mathrm{ext}}^{\perp}=0.6 \mathrm{~T}$ to screen the ground-state electron from the nuclear field fluctuations recovers high visibility TPI with a deconvolved (raw) fit value of $\nu=0.97 \pm 0.03$ $(\nu=0.64 \pm 0.06)$ [see Fig. 4(c)]. This near perfect indistinguishability meets the expectation based on the spectra in Fig. 1(e).

In summary, we have characterized the effect of nuclear field fluctuations on the RF spectrum and photon indistinguishability from $X^{0}$ and $X^{1-}$ in self-assembled InGaAs QDs. For the $X^{0}$, the large electron-hole exchange interaction relative to $B_{N}$ suppresses the effect of nuclear field fluctuations even at $B_{\mathrm{ext}}=0 \mathrm{~T}$. For a QD with a resident

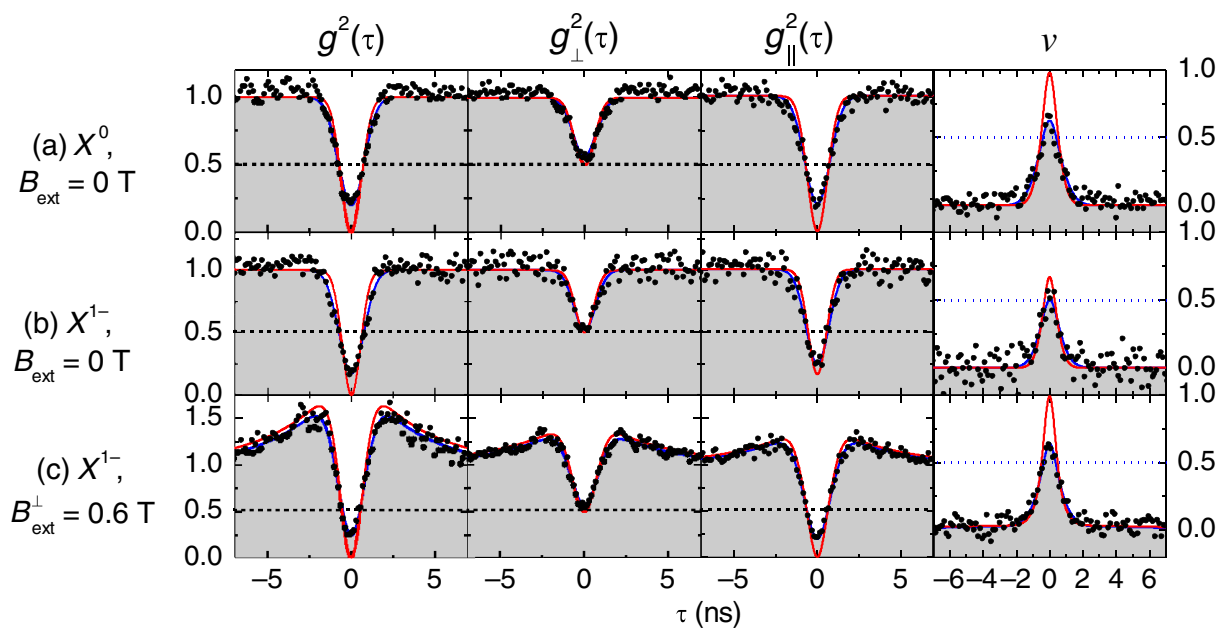

FIG. 4. Second-order correlation and two-photon interference measurements on (a) $X^{0}$, (b) $X^{1-}$ at $B_{\text {ext }}=0 \mathrm{~T}$, and (c) $X^{1-}$ at $B_{\text {ext }}^{\perp}=0.6 \mathrm{~T}$. Blue (red) solid lines are (deconvolved) fits to the data. Near perfect TPI visibility is observed for $X^{0}$ and $X^{1-}$ with a screening of nuclear field fluctuations. For $X^{1-}$ at $B_{\text {ext }}=0 \mathrm{~T}, \nu$ is reduced to $\approx 0.67$ due to $B_{N}$. Data are from sample 2 . 
electron in the ground state, the RF spectrum exhibits an inelastic doublet due to spin-flip Raman scattering, which reduces the TPI visibility. Application of $B_{\mathrm{ext}}^{\perp}$ screens the electron spin from $B_{N}^{\|}$, drastically decreasing the rate of Raman scattering and recovering near-ideal indistinguishability. An interesting prospect would be to investigate the positively charged exciton $X^{1+}$, for which the ground hole spin states can be more robust against nuclear spin noise at $B_{\text {ext }}=0 \mathrm{~T}$.

This work was supported by the EPSRC (Grants No. EP/ I023186/1, No. EP/K015338/1, and No. EP/G03673X/1) and by ERC Starting Grant No. 307392. B. D. G. thanks the Royal Society and E. M. G. thanks the Royal Society of Edinburgh for their personal research fellowships.

R. N. E. M. and T. S. S. contributed equally to this Letter.

*b.d.gerardot@hw.ac.uk

[1] J. L. O'Brien, A. Furusawa, and J. Vučković, Nat. Photonics 3, 687 (2009).

[2] S. Ates, M. Ulrich, S. Reitzenstein, A. Löffler, A. Forchel, and P. Michler, Phys. Rev. Lett. 103, 167402 (2009).

[3] C. Matthiesen, M. Geller, C. H. H. Schulte, C. L. Gall, J. Hansom, Z. Li, M. Hugues, E. Clarke, and M. Atatüre, Nat. Commun. 4, 1600 (2013).

[4] Y.-M. He, Y. He, Y.J. Wei, D. Wu, M. Atatüre, C. Schneider, S. Höfling, M. Kamp, C.-Y. Lu, and J.-W. Pan, Nat. Nanotechnol. 8, 213 (2013).

[5] L. Monniello, A. Reigue, R. Hostein, A. Lemaitre, A. Martinez, and R. Grousson, Phys. Rev. B 90, 041303(R) (2014).

[6] R. Proux, M. Maragkou, E. Baudin, C. Voisin, P. Roussignol, and C. Diederichs, Phys. Rev. Lett. 114, 067401 (2015).

[7] X. Ding et al., Phys. Rev. Lett. 116, 020401 (2016).

[8] N. Somaschi et al., Nat. Photonics 10, 340 (2016).

[9] J. C. Loredo et al., Optica 3, 433 (2016).

[10] A. C. Dada, T. S. Santana, R. N. E. Malein, Antonios Koutroumanis, Y. Ma, J. M. Zajac, J. Y. Lim, J. D. Song, and B. D. Gerardot, Optica 3, 493 (2016).

[11] B. R. Mollow, Phys. Rev. 188, 1969 (1969).

[12] H. S. Nguyen, G. Sallen, C. Voisin, P. Roussignol, C. Diederichs, and G. Cassabois, Appl. Phys. Lett. 99, 261904 (2011).

[13] C. Matthiesen, A. N. Vamivakus, and M. Atatüre, Phys. Rev. Lett. 108, 093602 (2012).

[14] K. Konthasinghe et al., Phys. Rev. B 85, 235315 (2012).

[15] A. Högele, S. Seidl, M. Kroner, K. Karrai, R. J. Warburton, B. D. Gerardot, and P. M. Petroff, Phys. Rev. Lett. 93, 217401 (2004).

[16] R. Loudon, The Quantum Theory of Light, 3rd ed., Oxford Science Publications (Oxford University Press, Oxford, 2000).

[17] A. V. Kuhlmann, J. Houel, A. Ludwig, L. Greuter, D. Reuter, A. D. Wieck, M. Poggio, and R. J. Warburton, Nat. Phys. 9, 570 (2013).

[18] G. Fernandez, T. Volz, R. Desbuquois, A. Badolato, and A. Imamoglu, Phys. Rev. Lett. 103, 087406 (2009).

[19] C. Santori, D. Fattal, K.-M. C Fu, P. E Barclay, and R. G Beausoleil, New J. Phys. 11, 123009 (2009).
[20] Y. He et al., Phys. Rev. Lett. 111, 237403 (2013).

[21] T. M. Sweeney, S. G. Carter, A. S. Bracker, M. Kim, C. S. Kim, L. Yang, P. M. Vora, P. G. Brereton, E. R. Cleveland, and D. Gammon, Nat. Photonics 8, 442 (2014).

[22] S. T. Yılmaz, P. Fallahi, and A. Imamoğlu, Phys. Rev. Lett. 105, 033601 (2010).

[23] K. De Greve et al., Nature (London) 491, 421 (2012).

[24] W. B. Gao, P. Fallahi, J. Miguel-Sanchez, and A. Imamoğlu, Nature (London) 491, 426 (2012).

[25] J. R. Schaibley, A. P. Burgers, G. A. McCracken, L.-M. Duan, P. R. Berman, D. G. Steel, A. S. Bracker, D. Gammon, and L. J. Sham, Phys. Rev. Lett. 110, 167401 (2013).

[26] A. Delteil, Z. Sun, W.-b. Gao, E. Togan, S. Faelt, and A. Imamoğlu, Nat. Phys. 12, 218 (2016).

[27] X. Xu, Y. Wu, B. Sun, Q. Huang, J. Cheng, D. G. Steel, A. S. Bracker, D. Gammon, C. Emary, and L. J. Sham, Phys. Rev. Lett. 99, 097401 (2007).

[28] I. A. Merkulov, A. L. Efros, and M. Rosen, Phys. Rev. B 65, 205309 (2002).

[29] B. Urbaszek, Xavier Marie, Thierry Amand, Olivier Krebs, Paul Voisin, Patrick Maletinsky, Alexander Högele, and Atac Imamoglu, Rev. Mod. Phys. 85, 79 (2013).

[30] P.-F. Braun et al., Phys. Rev. Lett. 94, 116601 (2005).

[31] A. V. Kuhlmann, J. H. Prechtel, J. Houel, A. Ludwig, D. Reuter, A. D. Wieck, and R. J. Warburton, Nat. Commun. 6, 8204 (2015).

[32] J. Hansom, C. H. H. Schulte, C. L. Gall, Clemens Matthiesen, E. Clarke, M. Hugues, J. M. Taylor, and M. Atatüre, Nat. Phys. 10, 725 (2014).

[33] M. Bayer et al., Phys. Rev. B 65, 195315 (2002).

[34] B. D. Gerardot, S. Seidl, P. A. Dalgarno, R. J. Warburton, M. Kroner, K. Karrai, A. Badolato, and P. M. Petroff, Appl. Phys. Lett. 90, 221106 (2007).

[35] Y. Ma, P. E. Kremer, and B. D. Gerardot, J. Appl. Phys. 115, 023106 (2014).

[36] A. Muller, E. B. Flagg, P. Bianucci, X. Y. Wang, D. G. Deppe, W. Ma, J. Zhang, G. J. Salama, M. Xiao, and C. K. Shih, Phys. Rev. Lett. 99, 187402 (2007).

[37] J. Fischer, W. A. Coish, D. V. Bulaev, and D. Loss, Phys. Rev. B 78, 155329 (2008).

[38] J. Houel, J. H. Prechtel, A. V. Kuhlmann, D. Brunner, C. E. Kuklewicz, B. D. Gerardot, N. G. Stoltz, P. M. Petroff, and R. J. Warburton, Phys. Rev. Lett. 112, 107401 (2014).

[39] See Supplemental Material at http://link.aps.org/ supplemental/10.1103/PhysRevLett.116.257401, which includes Refs. [40-44], for details on the experimental setup, determination of TPI visibility, and theoretical modelling of the $X^{1-}$ system.

[40] R. B. Patel, A. J. Bennett, K. Cooper, P. Atkinson, C. A. Nicoll, D. A. Ritchie, and A. J. Shields, Phys. Rev. Lett. 100, 207405 (2008).

[41] T. Legero, T. Wilk, A. Kuhn, and G. Rempe, Appl. Phys. B 77, 797 (2003)

[42] R. J. Warburton, Nat. Mater. 12, 483 (2013).

[43] P. Fallahi, S. T. Yılmaz, and A. Imamoğlu, Phys. Rev. Lett. 105, 257402 (2010).

[44] E. A. Chekhovich, M. N. Makhonin, K. V. Kavokin, A. B. Krysa, M. S. Skolnick, and A. I. Tartakovskii, Phys. Rev. Lett. 104, 066804 (2010).

[45] J. Dreiser, M. Atatüre, C. Galland, T. Müller, A. Badolato, and A. Imamoglu, Phys. Rev. B 77, 075317 (2008). 